\title{
CLOSED FORM EVALUATION OF MELHAM'S RECIPROCAL SUMS
}

\section{E. KILIÇ AND H. PRODINGER}

Received 13 June, 2014

\begin{abstract}
Recently Melham [1] gives closed formulæ for certain finite reciprocal sums. In this paper, we present a different approach to compute these sums in closed form. Our approach is straight-forward and simple. First we convert the sums in $q$-notation, then use partial fraction decomposition and telescoping to derive closed formulæ.
\end{abstract}

2010 Mathematics Subject Classification: 11B39

Keywords: reciprocal sums identities, partial fraction decomposition

\section{INTRODUCTION}

Let $a \geq 0$ and $b \geq 0$ be integers with $(a, b) \neq(0,0)$. For $p$ a positive integer, define the sequences $\left\{W_{n}\right\}$ and $\left\{\bar{W}_{n}\right\}$ by

$$
W_{n}=p W_{n-1}+W_{n-2} \text { and } \bar{W}_{n}=W_{n-1}+W_{n+1},
$$

where $W_{0}=a, W_{1}=b$.

When $(a, b, p)=(0,1,1)$, we have $\left\{W_{n}\right\}=\left\{F_{n}\right\}$, and $\left\{\bar{W}_{n}\right\}=\left\{L_{n}\right\}$, which are the Fibonacci and Lucas numbers, respectively. When $(a, b, p)=(0,1, p)$, we denote $\left\{W_{n}\right\}=\left\{U_{n}\right\}$, and $\left\{\bar{W}_{n}\right\}=\left\{V_{n}\right\}$, which are the generalized Fibonacci and Lucas numbers, respectively.

For $k \geq 1, m \geq 0$, and $n \geq 2$, the author [1] gave closed formulæ for the following finite reciprocal sums:

(1) For nonnegative integers $m_{1}<m_{2}$ and $m_{3}<m_{4}$ with $m_{1}+m_{2}=m_{3}+m_{4}$,

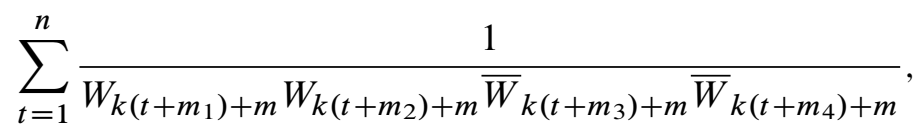

(2) For nonnegative integers $m_{i}$ with $m_{i}<m_{i+1}$ for $1<i<4$ and positive integer $c$,
i) $\sum_{t=1}^{n} \frac{U_{2 k t+2 m}}{P_{6}(W, \bar{W})}$
ii) $\sum_{t=1}^{n} \frac{U_{2 k\left(t+m_{1}\right)+2 m}}{P_{6}(W, \bar{W})}$
iii) $\sum_{t=1}^{n} \frac{U_{2 k\left(t+m_{2}\right)+2 m}}{P_{6}(W, \bar{W})}$

(c) 2017 Miskolc University Press 
(3)

(4)
i) $\sum_{t=1}^{n} \frac{1}{P_{8}(W, \bar{W})}$
ii) $\sum_{t=1}^{n} \frac{U_{2 k(t+c)+2 m} U_{2 k(t+2 c)+2 m}}{P_{8}(W, \bar{W}, c, 2 c, 3 c)}$,

i) $\sum_{t=1}^{n} \frac{U_{2 k\left(t+m_{1}\right)+2 m} U_{2 k\left(t+m_{2}\right)+2 m} U_{2 k\left(t+m_{3}\right)+2 m}}{P_{10}(W, \bar{W})}$ ii) $\sum_{t=1}^{n} \frac{1}{P_{12}(W, \bar{W})}$,

where $P_{2 r}\left(W, \bar{W}, m_{1}, m_{2}, \ldots, m_{r-1}\right)$, or briefly $P_{2 r}(W, \bar{W})$, is given by

$$
P_{2 r}(W, \bar{W})=W_{k t+m} \bar{W}_{k t+m} \prod_{i=1}^{r-1} W_{k\left(t+m_{i}\right)+m} \bar{W}_{k\left(t+m_{i}\right)+m} .
$$

In this paper, we present a different approach to compute these sums. Our approach is straight-forward and simple and works in all instances. First we convert these sums in $q$-notation and use partial fraction decomposition. Using telescoping, we derive closed formulæ for them.

The Binet forms are

$$
\begin{gathered}
W_{n}=\frac{A \alpha^{n}-B \beta^{n}}{\alpha-\beta}=A \alpha^{n-1} \frac{\left(1-q^{n} B / A\right)}{(1-q)}, \\
\bar{W}_{n}=A \alpha^{n}+B \beta^{n}=A \alpha^{n}\left(1+q^{n} B / A\right)
\end{gathered}
$$

and

$$
U_{n}=\frac{\alpha^{n}-\beta^{n}}{\alpha-\beta}=\alpha^{n-1} \frac{\left(1-q^{n}\right)}{(1-q)}, \quad V_{n}=\alpha^{n}+\beta^{n}=\alpha^{n}\left(1+q^{n}\right),
$$

where $\alpha, \beta=\left(p \mp \sqrt{p^{2}+4}\right) / 2, q=\beta / \alpha, \alpha=\mathbf{i} q^{-\frac{1}{2}}, A=b-a \beta$ and $B=b-a \alpha$.

We frequently denote the sequences $\left\{W_{n}\right\}$ and $\left\{\bar{W}_{n}\right\}$ by $\left\{W_{n}(A, B)\right\}$ and $\left\{\bar{W}_{n}(A, B)\right\}$, respectively.

\section{Simple evaluation of Melham's sums}

1. We start with the first kind of sums by converting them into $q$-notation:

$$
\begin{aligned}
& \sum_{t=1}^{n} \frac{1}{W_{k\left(t+m_{1}\right)+m} W_{k\left(t+m_{2}\right)+m} \bar{W}_{k\left(t+m_{3}\right)+m} \bar{W}_{k\left(t+m_{4}\right)+m}} \\
& =\frac{(1-q)^{2}}{A^{4} \alpha^{k\left(m_{1}+m_{2}+m_{3}+m_{4}\right)+4 m-2}} \\
& \times \sum_{t=1}^{n}\left(\frac{q^{2 k t}}{\left(1-q^{k\left(t+m_{1}\right)+m} B / A\right)\left(1-q^{k\left(t+m_{2}\right)+m} B / A\right)}\right. \\
& \left.\times \frac{1}{\left(1+q^{k\left(t+m_{3}\right)+m} B / A\right)\left(1+q^{k\left(t+m_{4}\right)+m} B / A\right)}\right),
\end{aligned}
$$


where $m_{1}, m_{2}, m_{3}$ and $m_{4}$ are defined as before.

Without constant factor and writing $z=q^{t k}$ and $q^{k m_{i}+m}=q^{c_{i}}$ and $B / A=a$ for $1 \leq i \leq 4$, we define

$$
S_{n}:=\sum_{t=1}^{n} \frac{z^{2}}{\left(1-a z q^{c_{1}}\right)\left(1-a z q^{c_{2}}\right)\left(1+a z q^{c_{3}}\right)\left(1+a z q^{c_{4}}\right)} .
$$

Let

$$
T(z):=\frac{z^{2}}{\left(1-a z q^{c_{1}}\right)\left(1-a z q^{c_{2}}\right)\left(1+a z q^{c_{3}}\right)\left(1+a z q^{c_{4}}\right)} .
$$

The partial fraction decomposition of $T(z)$ is

$$
\begin{aligned}
& \frac{z^{2}}{\left(1-a z q^{c_{1}}\right)\left(1-a z q^{c_{2}}\right)\left(1+a z q^{c_{3}}\right)\left(1+a z q^{c_{4}}\right)} \\
& =-\frac{q^{c_{4}}}{a^{2}\left(q^{c_{2}}+q^{c_{4}}\right)\left(q^{c_{1}}+q^{c_{4}}\right)\left(q^{c_{3}}-q^{c_{4}}\right)\left(1+a q^{c_{4}} z\right)} \\
& -\frac{q^{c_{2}}}{a^{2}\left(q^{c_{2}}+q^{c_{3}}\right)\left(q^{c_{2}}+q^{c_{4}}\right)\left(q^{c_{1}}-q^{c_{2}}\right)\left(1-a q^{c_{2}} z\right)} \\
& +\frac{q^{c_{1}}}{a^{2}\left(q^{c_{1}}+q^{c_{4}}\right)\left(q^{c_{1}}+q^{c_{3}}\right)\left(q^{c_{1}}-q^{c_{2}}\right)\left(1-a q^{c_{1}} z\right)} \\
& +\frac{q^{c_{3}}}{a^{2}\left(q^{c_{3}}-q^{c_{4}}\right)\left(q^{c_{2}}+q^{c_{3}}\right)\left(q^{c_{1}}+q^{c_{3}}\right)\left(1+a q^{c_{3}} z\right)} .
\end{aligned}
$$

The assumption $m_{1}<m_{2}$ and $m_{3}<m_{4}$ with $m_{1}+m_{2}=m_{3}+m_{4}$ means $c_{1}<c_{2}$ and $c_{3}<c_{4}$ with $c_{1}+c_{2}=c_{3}+c_{4}$. We write

$$
\begin{aligned}
& \frac{z^{2}}{\left(1-a z q^{c_{1}}\right)\left(1-a z q^{c_{2}}\right)\left(1+a z q^{c_{3}}\right)\left(1+a z q^{c_{4}}\right)} \\
& \times a^{2}\left(q^{c_{2}}+q^{c_{4}}\right)\left(q^{c_{1}}+q^{c_{4}}\right)\left(q^{c_{3}}-q^{c_{4}}\right)\left(q^{c_{2}}+q^{c_{3}}\right)\left(q^{c_{1}}-q^{c_{2}}\right)\left(q^{c_{1}}+q^{c_{3}}\right) \\
= & -q^{c_{3}}\left(q^{c_{2}}+q^{c_{4}}\right)\left(q^{c_{1}}+q^{c_{4}}\right)\left(q^{c_{1}}-q^{c_{2}}\right)\left[\frac{1}{1+a q^{c_{4}} z}-\frac{1}{1+a q^{c_{3}} z}\right] \\
& -q^{c_{2}}\left(q^{c_{1}}+q^{c_{4}}\right)\left(q^{c_{3}}-q^{c_{4}}\right)\left(q^{c_{1}}+q^{c_{3}}\right)\left[\frac{1}{1-a q^{c_{2}} z}-\frac{1}{1-a q^{c_{1}} z}\right]
\end{aligned}
$$

and so

$$
\begin{aligned}
& a^{2} q^{c_{1}+c_{2}}\left(1+q^{c_{4}-c_{2}}\right)\left(1-q^{c_{4}-c_{3}}\right)\left(1+q^{c_{3}-c_{2}}\right)\left(1-q^{c_{2}-c_{1}}\right)\left(1+q^{c_{3}-c_{1}}\right) S_{n} \\
& =-\left(1+q^{c_{4}-c_{2}}\right)\left(1-q^{c_{2}-c_{1}}\right)\left[\sum_{t=1}^{n} \frac{1}{1+a q^{c_{3}+d} z}-\sum_{t=1}^{n} \frac{1}{1+a q^{c_{3}} z}\right] \\
& \left.-\left(1-q^{c_{4}-c_{3}}\right) 1+q^{c_{3}-c_{1}}\right)\left[\sum_{t=1}^{n} \frac{1}{1-a q^{c_{1}+c_{z}} z}-\sum_{t=1}^{n} \frac{1}{1-a q^{c_{1}} z}\right],
\end{aligned}
$$


which, by the telescoping sum identity

$$
\sum_{t=1}^{n}\left(\frac{1}{1+a z q^{b+c}}-\frac{1}{1+a z q^{c}}\right)=\sum_{t=1}^{b}\left(\frac{1}{1+a z q^{n+c}}-\frac{1}{1+a z q^{c}}\right)
$$

gives us

$$
\begin{aligned}
& a^{2} q^{c_{1}+c_{2}}\left(1+q^{c_{4}-c_{2}}\right)\left(1-q^{c_{4}-c_{3}}\right)\left(1+q^{c_{3}-c_{2}}\right)\left(1-q^{c_{2}-c_{1}}\right)\left(1+q^{c_{3}-c_{1}}\right) S_{n} \\
& =-\left(1+q^{c_{4}-c_{2}}\right)\left(1-q^{c_{2}-c_{1}}\right) \sum_{t=1}^{c_{4}-c_{3}}\left(\frac{1}{1+a z q^{n+c_{3}}}-\frac{1}{1+a z q^{c_{3}}}\right) \\
& -\left(1-q^{c_{4}-c_{3}}\right)\left(1+q^{c_{3}-c_{1}}\right) \sum_{t=1}^{c_{2}-c_{1}}\left(\frac{1}{1-a z q^{n+c_{1}}}-\frac{1}{1-a z q^{c_{1}}}\right) .
\end{aligned}
$$

We write it in original form

$$
\begin{aligned}
& (B / A)^{2} q^{k\left(m_{1}+m_{2}\right)+2 m}\left(1+q^{k\left(m_{4}-m_{2}\right)}\right)\left(1-q^{k\left(m_{4}-m_{3}\right)}\right) \\
& \times\left(1+q^{k\left(m_{3}-m_{2}\right)}\right)\left(1-q^{k\left(m_{2}-m_{1}\right)}\right)\left(1+q^{k\left(m_{3}-m_{1}\right)}\right) S_{n} \\
& =-\left(1+q^{k\left(m_{4}-m_{2}\right)}\right)\left(1-q^{k\left(m_{2}-m_{1}\right)}\right) \\
& \times \sum_{t=1}^{k\left(m_{4}-m_{3}\right)}\left(\frac{1}{1+q^{t k+n+k m_{3}+m} B / A}-\frac{1}{1+q^{t k+k m_{3}+m} B / A}\right) \\
& -\left(1-q^{k\left(m_{4}-m_{3}\right)}\right)\left(1+q^{k\left(m_{3}-m_{1}\right)}\right) \\
& \times \sum_{t=1}^{k\left(m_{2}-m_{1}\right)}\left(\frac{1}{1-q^{t k+n+k m_{1}+m} B / A}-\frac{1}{1-q^{t k+k m_{1}+m} B / A}\right)
\end{aligned}
$$

or

$$
\begin{aligned}
& S_{n}= \frac{A^{3} B^{-2} \alpha^{2\left(2 m+k m_{1}+k m_{2}\right)}}{\Delta^{2} V_{k\left(m_{4}-m_{2}\right)} V_{k\left(m_{3}-m_{1}\right)} V_{k\left(m_{3}-m_{2}\right)} U_{k\left(m_{2}-m_{1}\right)} U_{k\left(m_{4}-m_{3}\right)}} \\
& \times\left[\Delta V_{k\left(m_{4}-m_{2}\right)} U_{k\left(m_{2}-m_{1}\right)} \sum_{t=1}^{k\left(m_{4}-m_{3}\right)}\left(\frac{\alpha^{t k+n+k m_{3}+m}}{\bar{W}_{t+n+k m_{3}+m}}-\frac{\alpha^{t k+k m_{3}+m}}{\bar{W}_{t k+k m_{3}+m}}\right)\right. \\
&\left.\quad+U_{k\left(m_{4}-m_{3}\right)} V_{k\left(m_{3}-m_{1}\right)} \sum_{t=1}^{k\left(m_{2}-m_{1}\right)}\left(\frac{\alpha^{t k+n+k m_{1}+m}}{W_{t k+n+k m_{1}+m}}-\frac{\alpha^{t k+k m_{1}+m}}{W_{t k+k m_{1}+m}}\right)\right]
\end{aligned}
$$

and so

$$
\sum_{t=1}^{n} \frac{1}{W_{k\left(t+m_{1}\right)+m} W_{k\left(t+m_{2}\right)+m} \bar{W}_{k\left(t+m_{3}\right)+m} \bar{W}_{k\left(t+m_{4}\right)+m}}
$$




$$
\begin{aligned}
& =\frac{1}{A B^{2} V_{k\left(m_{4}-m_{2}\right)} V_{k\left(m_{3}-m_{1}\right)} V_{k\left(m_{3}-m_{2}\right)} U_{k\left(m_{2}-m_{1}\right)} U_{k\left(m_{4}-m_{3}\right)}} \\
& \times\left[\Delta V_{k\left(m_{4}-m_{2}\right)} U_{k\left(m_{2}-m_{1}\right)} \sum_{t=1}^{k\left(m_{4}-m_{3}\right)}\left(\frac{\alpha^{t k+n+k m_{3}+m}}{\bar{W}_{t+n+k m_{3}+m}}-\frac{\alpha^{t k+k m_{3}+m}}{\bar{W}_{t k+k m_{3}+m}}\right)\right. \\
& \left.+U_{k\left(m_{4}-m_{3}\right)} V_{k\left(m_{3}-m_{1}\right)} \sum_{t=1}^{k\left(m_{2}-m_{1}\right)}\left(\frac{\alpha^{t k+n+k m_{1}+m}}{W_{t k+n+k m_{1}+m}}-\frac{\alpha^{t k+k m_{1}+m}}{W_{t k+k m_{1}+m}}\right)\right],
\end{aligned}
$$

which is the desired evaluation of the first type of sums.

For example, we have

$$
\sum_{t=1}^{n} \frac{1}{F_{t+1} F_{t+2} L_{t+1} L_{t+2}}=-\frac{1}{2}\left[\sqrt{5}\left(\frac{\alpha^{n+2}}{L_{n+2}}-\frac{\alpha^{2}}{L_{2}}\right)+\left(\frac{\alpha^{n+2}}{F_{n+2}}-\alpha^{2}\right)\right]
$$

and

$$
\sum_{t=1}^{n} \frac{1}{F_{t} F_{t+3} L_{t+1} L_{t+2}}=-\frac{1}{6}\left[2 \sqrt{5}\left(\frac{\alpha^{n+2}}{L_{n+2}}-\frac{\alpha^{2}}{3}\right)-\sum_{t=1}^{3}\left(\frac{\alpha^{t+n}}{F_{t+n}}-\frac{\alpha^{t}}{F_{t}}\right)\right] .
$$

2. i) The sums take in $q$-notation the form

$$
\begin{aligned}
\sum_{t=1}^{n} \frac{U_{2 k t+2 m}}{P_{6}(W, \bar{W})}=A^{-6} & \alpha^{5-2 k m_{1}-2 k m_{2}-4 m}(1-q)^{2} \\
& \times \sum_{t=1}^{n}\left(\frac{\alpha^{-4 k t}\left(1-q^{2 k\left(t+m_{2}\right)+2 m}\right)}{\left(1-q^{2 k t+2 m}(B / A)^{2}\right)}\right. \\
& \left.\frac{1}{\left(1-q^{2 k\left(t+m_{1}\right)+2 m}(B / A)^{2}\right)\left(1-q^{2 k\left(t+m_{2}\right)+2 m}(B / A)^{2}\right)}\right) .
\end{aligned}
$$

Without constant factor and writing $z=q^{2 t k}, q^{2 k m_{i}}=q^{c_{i}}, q^{2 m}=c$ and $(B / A)^{2}=a$ for $1 \leq i \leq 2$, we consider

$$
S_{n}:=\sum_{t=1}^{n} \frac{z(1-c z)}{(1-a c z)\left(1-a c z q^{c_{1}}\right)\left(1-a c z q^{c_{2}}\right)} .
$$

Let

$$
T(z):=\frac{z(1-c z)}{(1-a c z)\left(1-a c z q^{c_{1}}\right)\left(1-a c z q^{c_{2}}\right)} .
$$

The partial fraction decomposition of $T(z)$ is

$$
\begin{aligned}
& T(z)=\frac{z(1-c z)}{(1-a c z)\left(1-a c z q^{c_{1}}\right)\left(1-a c z q^{c_{2}}\right)} \\
& =-\frac{(1-a)}{a^{2} c(1-a c z)\left(1-q^{c_{1}}\right)\left(1-q^{c_{2}}\right)}
\end{aligned}
$$




$$
\begin{aligned}
& -\frac{\left(1-a q^{c_{2}}\right)}{a^{2} c q^{c_{1}}\left(1-a c q^{c_{2}} z\right)\left(1-q^{c_{2}}\right)\left(1-q^{c_{2}-c_{1}}\right)} \\
& +\frac{\left(1-a q^{c_{1}}\right)}{a^{2} c q^{c_{1}}\left(1-q^{c_{1}}\right)\left(1-a c q^{c_{1}} z\right)\left(1-q^{c_{2}-c_{1}}\right)} .
\end{aligned}
$$

The assumption $0<m_{1}<m_{2}$ means $c_{1}<c_{2}$. By telescoping, we write

$$
\begin{aligned}
S_{n} & =\frac{(1-a)}{a^{2} c\left(1-q^{c_{1}}\right)\left(1-q^{c_{2}}\right)} \sum_{t=1}^{c_{1}}\left(\frac{1}{1-a c q^{n} z}-\frac{1}{1-a c z}\right) \\
& -\frac{\left(1-a q^{c_{2}}\right)}{a^{2} c q^{c_{1}}\left(1-q^{c_{2}}\right)\left(1-q^{c_{2}-c_{1}}\right)} \sum_{t=1}^{c_{2}-c_{1}}\left(\frac{1}{1-a c z q^{n+c_{1}}}-\frac{1}{1-a c z q^{c_{1}}}\right)
\end{aligned}
$$

and so we obtain

$$
\begin{aligned}
& \sum_{t=1}^{n} \frac{U_{2 k t+2 m}}{P_{6}(W, \bar{W})}=\frac{\left(A^{2}-B^{2}\right)}{A^{2} B^{4} \Delta U_{2 k m_{1}} U_{2 k m_{2}}} \\
& \times \sum_{t=1}^{2 k m_{1}}\left(\frac{\alpha^{2 t k+n+2 m+3}}{W_{2 t k+n+2 m}\left(A^{2}, B^{2}\right)}-\frac{\alpha^{2 t k+2 m+3}}{W_{2 t k+2 m}\left(A^{2}, B^{2}\right)}\right) \\
& -\frac{W_{2 k m_{2}}\left(A^{2}, B^{2}\right)}{A^{2} B^{4} U_{2 k m_{2}} U_{2 k\left(m_{2}-m_{1}\right)}} \\
& \times \sum_{t=1}^{2 k\left(m_{2}-m_{1}\right)}\left(\frac{\alpha^{2 t k+n+2 k m_{1}+2 m+3}}{W_{2 t k+n+2 k m_{1}+2 m}\left(A^{2}, B^{2}\right)}-\frac{\alpha^{2 t k+2 k m_{1}+2 m+3}}{W_{2 t k+2 k m_{1}+2 m}\left(A^{2}, B^{2}\right)}\right),
\end{aligned}
$$

where $\Delta$ is defined as before.

ii) Now we write the second class of sums in $q$-notation as

$$
\begin{aligned}
& \sum_{t=1}^{n} \frac{U_{2 k\left(t+m_{1}\right)+2 m}}{P_{6}(W, \bar{W})}=A^{-6} \alpha^{5-2 k m_{2}-4 m}(1-q)^{2} \\
& \times \sum_{t=1}^{n}\left(\frac{q^{2 k t}\left(1-q^{2 k\left(t+m_{1}\right)+2 m}\right)}{\left(1-q^{2 k t+2 m}(B / A)^{2}\right)\left(1-q^{2 k\left(t+m_{1}\right)+2 m}(B / A)^{2}\right)}\right) \\
& \left.\times \frac{1}{\left(1-q^{2 k\left(t+m_{2}\right)+2 m}(B / A)^{2}\right)}\right) .
\end{aligned}
$$

Without constant factor and writing $z=q^{2 t k}, q^{2 k m_{i}}=q^{c_{i}}$ and $(B / A)^{2}=a$ for $1 \leq i \leq 2$, we define the sum

$$
S_{n}:=\sum_{t=1}^{n} \frac{z\left(1-c z q^{c_{1}}\right)}{(1-a c z)\left(1-a c z q^{c_{1}}\right)\left(1-a c z q^{c_{2}}\right)} .
$$


Let

$$
T(z):=\frac{z\left(1-c z q^{c_{1}}\right)}{(1-a c z)\left(1-a c z q^{c_{1}}\right)\left(1-a c z q^{c_{2}}\right)} .
$$

Similarly as before, by using the partial fraction decomposition of $T(z)$ and telescoping, since $c_{2}>c_{1}$, we obtain

$$
\begin{aligned}
S_{n}=\frac{1}{a^{2} c\left(1-q^{c_{2}-c_{1}}\right)}\left[\frac{1-a}{1-q^{c_{1}}} \sum_{t=1}^{c_{1}}\right. & \left(\frac{1}{1-a c z q^{n}}-\frac{1}{1-a c z}\right) \\
& \left.-\frac{1-a q^{c_{2}-c_{1}}}{1-q^{c_{2}}} \sum_{t=1}^{c_{2}}\left(\frac{1}{1-a c z q^{n}}-\frac{1}{1-a c z}\right)\right] .
\end{aligned}
$$

Thus we get the result

$$
\begin{aligned}
& \sum_{t=1}^{n} \frac{U_{2 k\left(t+m_{1}\right)+2 m}}{P_{6}(W, \bar{W})}=\frac{\alpha^{2 m+3}}{B^{4} A^{2} U_{2 k\left(m_{2}-m_{1}\right)}} \\
& \times\left[\frac{\left(A^{2}-B^{2}\right)}{\Delta U_{2 k m_{1}}} \sum_{t=1}^{2 k m_{1}}\left(\frac{\alpha^{2 k t+n}}{W_{2 t k+2 m+n}\left(A^{2}, B^{2}\right)}-\frac{\alpha^{2 k t}}{W_{2 t k+2 m}\left(A^{2}, B^{2}\right)}\right)\right. \\
& \left.-\frac{W_{2 k\left(m_{2}-m_{1}\right)}\left(A^{2}, B^{2}\right)}{U_{2 k m_{2}}} \sum_{t=1}^{2 k m_{2}}\left(\frac{\alpha^{2 k t+n}}{W_{2 t k+2 m+n}\left(A^{2}, B^{2}\right)}-\frac{\alpha^{2 k t}}{W_{2 t k+2 m}\left(A^{2}, B^{2}\right)}\right)\right],
\end{aligned}
$$

where $\Delta$ is defined as before.

For the next sums, we only present the key steps, as the procedure is always the same.

iii) Consider the sums and its $q$-form

$$
\begin{aligned}
& \sum_{t=1}^{n} \frac{U_{2 k\left(t+m_{2}\right)+2 m}}{P_{6}(W, \bar{W})}=A^{-6} \alpha^{5-2 k m_{1}-4 m}(1-q)^{2} \\
& \times \sum_{t=1}^{n}\left(\frac{q^{2 k t}\left(1-q^{2 k\left(t+m_{2}\right)+2 m}\right)}{\left(1-q^{2 k t+2 m}(B / A)^{2}\right)\left(1-q^{2 k\left(t+m_{1}\right)+2 m}(B / A)^{2}\right)}\right. \\
& \left.\times \frac{1}{\left(1-q^{2 k\left(t+m_{2}\right)+2 m}(B / A)^{2}\right)}\right) .
\end{aligned}
$$

Without constant factor and rewriting the parameters, we consider the sums

$$
S_{n}:=\sum_{t=1}^{n} \frac{z\left(1-c z q^{c_{2}}\right)}{(1-a c z)\left(1-a c z q^{c_{1}}\right)\left(1-a c z q^{c_{2}}\right)} .
$$

Here by the partial fraction decomposition 


$$
\begin{gathered}
\frac{z\left(1-c z q^{c_{2}}\right)}{(1-a c z)\left(1-a c z q^{c_{1}}\right)\left(1-a c z q^{c_{2}}\right)}=\frac{1}{a^{2} c}\left(\frac{\left(a-q^{c_{2}}\right)}{(1-a c z)\left(1-q^{c_{1}}\right)\left(1-q^{c_{2}}\right)}\right. \\
\left.-\frac{q^{c_{2}-c_{1}}(1-a)}{\left(1-a c q^{c_{2}} z\right)\left(1-q^{c_{2}}\right)\left(1-q^{c_{2}-c_{1}}\right)}-\frac{\left(a-q^{c_{2}-c_{1}}\right)}{\left(1-q^{c_{1}}\right)\left(1-a c q^{c_{1}} z\right)\left(1-q^{c_{2}-c_{1}}\right)}\right),
\end{gathered}
$$

and using telescoping, since $c_{2}>c_{1}$, we write

$$
\begin{aligned}
S_{n}=-\frac{1}{a^{2} c\left(1-q^{c_{2}-c_{1}}\right)}[ & \frac{a\left(1-q^{c_{2}-c_{1}} a^{-1}\right)}{1-q^{c_{1}}} \sum_{t=1}^{c_{1}}\left(\frac{1}{1-a c z q^{n}}-\frac{1}{1-a c z}\right) \\
& \left.+\frac{q^{c_{2}-c_{1}}(1-a)}{1-q^{c_{2}}} \sum_{t=1}^{c_{2}}\left(\frac{1}{1-a c z q^{n}}-\frac{1}{1-a c z}\right)\right]
\end{aligned}
$$

and obtain the result

$$
\begin{aligned}
& \sum_{t=1}^{n} \frac{U_{2 k\left(t+m_{2}\right)+2 m}}{P_{6}(W, \bar{W})}=-\frac{1}{B^{4} A^{2} U_{2 k\left(m_{2}-m_{1}\right)}} \\
& \times\left[\frac{W_{2 k\left(m_{2}-m_{1}\right)}\left(B^{2}, A^{2}\right)}{U_{2 k m_{1}}} \sum_{t=1}^{2 k m_{1}}\left(\frac{\alpha^{2 k t+2 m+n+5}}{W_{2 k t+2 m+n}\left(A^{2}, B^{2}\right)}-\frac{\alpha^{2 k t+2 m+5}}{W_{2 k t+2 m}\left(A^{2}, B^{2}\right)}\right)\right. \\
& \left.\quad+\frac{\left(A^{2}-B^{2}\right)}{(\alpha-\beta) U_{2 k m_{2}}} \sum_{t=1}^{2 k m_{2}}\left(\frac{\alpha^{2 k t+2 m+n+3}}{W_{2 k t+2 m+n}\left(A^{2}, B^{2}\right)}-\frac{\alpha^{2 k t+2 m+3}}{W_{2 k t+2 m}\left(A^{2}, B^{2}\right)}\right)\right] .
\end{aligned}
$$

3. i) For $0<m_{1}<m_{2}<m_{3}$, we consider the following sums in its $q$-form

$$
\begin{aligned}
\sum_{t=1}^{n} \frac{1}{P_{8}(W, \bar{W})} & =A^{-8} \alpha^{8-2 k m_{1}-2 k m_{2}-2 k m_{3}-8 m}(1-q)^{4} \\
& \times \sum_{t=1}^{n} \frac{z^{2}}{(1-a c z)\left(1-a c z q^{c_{1}}\right)\left(1-a c z q^{c_{2}}\right)\left(1-a c z q^{c_{3}}\right)} .
\end{aligned}
$$

Without constant factor, we only consider the sum

$$
S_{n}:=\sum_{t=1}^{n} \frac{z^{2}}{(1-a c z)\left(1-a c z q^{c_{1}}\right)\left(1-a c z q^{c_{2}}\right)\left(1-a c z q^{c_{3}}\right)} .
$$

By partial fraction decomposition of the summand and using telescoping, we write

$$
\begin{aligned}
& a^{2} c^{2} q^{c_{1}+c_{2}}\left(1-q^{c_{1}}\right)\left(1-q^{c_{2}}\right)\left(1-q^{c_{3}}\right)\left(1-q^{c_{3}-c_{2}}\right)\left(1-q^{c_{2}-c_{1}}\right)\left(1-q^{c_{3}-c_{1}}\right) S_{n} \\
& =q^{c_{3}}\left(1-q^{c_{1}+c_{2}-c_{3}}\right)\left(1-q^{c_{3}}\right)\left(1-q^{c_{2}-c_{1}}\right) \sum_{t=1}^{c_{2}}\left(\frac{1}{1-a c q^{n} z}-\frac{1}{1-a c z}\right) \\
& -q^{c_{3}}\left(1-q^{c_{2}-c_{1}}\right)\left(1-q^{c_{1}}\right)\left(1-q^{c_{2}}\right) \sum_{t=1}^{c_{3}}\left(\frac{1}{1-a c q^{n} z}-\frac{1}{1-a c z}\right)
\end{aligned}
$$




$$
+q^{c_{2}}\left(1-q^{c_{3}-c_{2}}\right)\left(1-q^{c_{2}}\right)\left(1-q^{c_{3}}\right) \sum_{t=1}^{c_{2}-c_{1}}\left(\frac{1}{1-a c q^{n+c_{1} z}}-\frac{1}{1-a c q^{c_{1} z}}\right) .
$$

Consequently after converting it back into the original form,

$$
\begin{aligned}
& \sum_{t=1}^{n} \frac{1}{P_{8}(W, \bar{W})} \\
& =\frac{\alpha^{2 m+4}}{A^{2} B^{4} U_{2 k m_{1}} U_{2 k m_{2}} U_{2 k m_{3}} U_{2 k\left(m_{3}-m_{2}\right)} U_{2 k\left(m_{2}-m_{1}\right)} U_{2 k\left(m_{3}-m_{1}\right)}} \\
& \times\left[U_{2 k\left(m_{1}+m_{2}-m_{3}\right)} U_{2 k m_{3}} U_{2 k\left(m_{2}-m_{1}\right)}\right. \\
& \times \sum_{t=1}^{2 k m_{2}}\left(\frac{\alpha^{n+2 t k}}{W_{n+2 t k+2 m}\left(A^{2}, B^{2}\right)}-\frac{\alpha^{2 t k}}{W_{2 t k+2 m}\left(A^{2}, B^{2}\right)}\right) \\
& -U_{2 k\left(m_{2}-m_{1}\right)} U_{2 k m_{1}} U_{2 k m_{2}} \sum_{t=1}^{2 k m_{3}}\left(\frac{\alpha^{n+2 t k}}{W_{n+2 t k+2 m}\left(A^{2}, B^{2}\right)}-\frac{\alpha^{2 t k}}{W_{2 t k+2 m}\left(A^{2}, B^{2}\right)}\right) \\
& +U_{2 k\left(m_{3}-m_{2}\right)} U_{2 k m_{2}} U_{2 k m_{3}} \\
& \left.\quad \times \sum_{t=1}^{2 k\left(m_{2}-m_{1}\right)}\left(\frac{\alpha^{n+2 t k+2 k m_{1}}}{W_{n+2 t k+2 k m_{1}+2 m}\left(A^{2}, B^{2}\right)}-\frac{\alpha^{2 t k+2 k m_{1}}}{W_{2 t k+2 k m_{1}+2 m}\left(A^{2}, B^{2}\right)}\right)\right] .
\end{aligned}
$$

3. ii) We now consider the sums

$$
\sum_{t=1}^{n} \frac{U_{2 k(t+g)+2 m} U_{2 k(t+2 g)+2 m}}{P_{8}(W, \bar{W}, g, 2 g, 3 g)} .
$$

Converting it into $q$-form and rewriting the parameters, that is, $z=q^{2 t k}, q^{2 m}=c$, $q^{2 k g}=q^{d}$ and $(B / A)^{2}=a$, we get

$$
A^{-8} \alpha^{6-6 g k-4 m}(1-q)^{2} \sum_{t=1}^{n} \frac{z\left(1-c z q^{d}\right)\left(1-c z q^{2 d}\right)}{(1-a c z)\left(1-a c z q^{d}\right)\left(1-a c z q^{2 d}\right)\left(1-a c z q^{3 d}\right)} .
$$

Without constant factor, we consider

$$
S_{n}:=\sum_{t=1}^{n} \frac{z\left(1-c z q^{d}\right)\left(1-c z q^{2 d}\right)}{(1-a c z)\left(1-a c z q^{d}\right)\left(1-a c z q^{2 d}\right)\left(1-a c z q^{3 d}\right)} .
$$

By partial fraction decomposition of the summand of $S_{n}$ and using telescoping, we obtain

$$
S_{n}=\frac{1}{a^{3} c\left(1-q^{d}\right)^{2}\left(1-q^{2 d}\right)}
$$




$$
\begin{aligned}
& \times\left[\left(a-q^{d}\right)(1-a)\left(1-q^{3 d}\right) \sum_{t=1}^{d}\left(\frac{1}{1-a c z q^{n}}-\frac{1}{1-a c z}\right)\right. \\
& -\left(1-a q^{d}\right)\left(1-a q^{2 d}\right)\left(1-q^{d}\right) \sum_{t=1}^{3 d}\left(\frac{1}{1-a c z q^{n}}-\frac{1}{1-a c z}\right) \\
& \left.+\left(1-a q^{d}\right)(1-a)\left(1-q^{3 d}\right) \sum_{t=1}^{2 d}\left(\frac{1}{1-a c z q^{n}}-\frac{1}{1-a c z}\right)\right] .
\end{aligned}
$$

Thus we get

$$
\begin{aligned}
& \sum_{t=1}^{n} \frac{U_{2 k(t+g)+2 m} U_{2 k(t+2 g)+2 m}}{P_{8}(W, \bar{W}, g, 2 g, 3 g)} \\
& =\frac{1}{B^{6} A^{4} U_{2 k g}^{2} U_{4 k g}}\left[W_{2 k g}\left(B^{2}, A^{2}\right)\left(A^{2}-B^{2}\right) U_{6 k g}\right. \\
& \times \sum_{t=1}^{d}\left(\frac{\alpha^{2 m+n-6 g k+2 k t+4}}{W_{n+2 k t+2 m}\left(A^{2}, B^{2}\right)}-\frac{\alpha^{2 m-6 g k+2 k t+4}}{W_{2 k t+2 m}\left(A^{2}, B^{2}\right)}\right) \\
& -\Delta W_{2 k g}\left(A^{2}, B^{2}\right) W_{4 k g}\left(A^{2}, B^{2}\right) U_{2 k g} \\
& \times \sum_{t=1}^{3 d}\left(\frac{\alpha^{2 m+n-6 g k+2 k t+4}}{W_{n+2 k t+2 m}\left(A^{2}, B^{2}\right)}-\frac{\alpha^{2 m-6 g k+2 k t+4}}{W_{2 k t+2 m}\left(A^{2}, B^{2}\right)}\right) \\
& +W_{2 k g}\left(A^{2}, B^{2}\right)\left(A^{2}-B^{2}\right) U_{6 k g} \\
& \left.\times \sum_{t=1}^{2 d}\left(\frac{\alpha^{2 m+n-6 g k+2 k t+4}}{W_{n+2 k t+2 m}\left(A^{2}, B^{2}\right)}-\frac{\alpha^{2 m-6 g k+2 k t+4}}{W_{2 k t+2 m}\left(A^{2}, B^{2}\right)}\right)\right]
\end{aligned}
$$

where $\Delta$ is defined as before.

4. i) For $0<m_{1}<m_{2}<m_{3}<m_{4}$, we consider the sums

$$
\sum_{t=1}^{n} \frac{U_{2 k\left(t+m_{1}\right)+2 m} U_{2 k\left(t+m_{2}\right)+2 m} U_{2 k\left(t+m_{3}\right)+2 m}}{P_{10}(W, \bar{W})} .
$$

If we convert it into $q$-notation and write $z=q^{2 t k}, q^{2 m}=c, q^{2 k m_{i}}=q^{c_{i}}$ and $(B / A)^{2}=a$ for $1 \leq i \leq 4$, then the sums above equals

$$
\begin{aligned}
& A^{-10} \alpha^{7-2 k m_{4}-4 m}(1-q)^{2} \\
& \times \sum_{t=1}^{n} \frac{z\left(1-c z q^{c_{1}}\right)\left(1-c z q^{c_{2}}\right)\left(1-c z q^{c_{3}}\right)}{(1-a c z)\left(1-a c z q^{c_{1}}\right)\left(1-a c z q^{c_{2}}\right)\left(1-a c z q^{c_{3}}\right)\left(1-a c z q^{c_{4}}\right)} .
\end{aligned}
$$


Without constant factor, we consider the sums

$$
S_{n}:=\sum_{t=1}^{n} \frac{z\left(1-c z q^{c_{1}}\right)\left(1-c z q^{c_{2}}\right)\left(1-c z q^{c_{3}}\right)}{(1-a c z)\left(1-a c z q^{c_{1}}\right)\left(1-a c z q^{c_{2}}\right)\left(1-a c z q^{c_{3}}\right)\left(1-a c z q^{c_{4}}\right)} .
$$

The partial fraction decomposition of the summand is

$$
\begin{aligned}
& \frac{z\left(1-c z q^{c_{1}}\right)\left(1-c z q^{c_{2}}\right)\left(1-c z q^{c_{3}}\right) a^{4} c}{(1-a c z)\left(1-a c z q^{c_{1}}\right)\left(1-a c z q^{c_{2}}\right)\left(1-a c z q^{c_{3}}\right)\left(1-a c z q^{c_{4}}\right)} \\
& =B\left(\frac{1}{1-a c q^{c_{1}} z}-\frac{1}{1-a c z}\right)-C\left(\frac{1}{1-a c q^{c_{2}} z}-\frac{1}{1-a c z}\right) \\
& +D\left(\frac{1}{1-a c q^{c_{3}} z}-\frac{1}{1-a c z}\right)-E\left(\frac{1}{1-a c q^{c_{4}} z}-\frac{1}{1-a c z}\right),
\end{aligned}
$$

where

$$
\begin{aligned}
& A=\frac{a^{3}\left(1-a^{-1} q^{c_{1}}\right)\left(1-a^{-1} q^{c_{2}}\right)\left(1-a^{-1} q^{c_{3}}\right)}{\left(1-q^{c_{1}}\right)\left(1-q^{c_{2}}\right)\left(1-q^{c_{3}}\right)\left(1-q^{c_{4}}\right)}, \\
& B=\frac{a^{2}\left(1-a^{-1} q^{c_{2}-c_{1}}\right)\left(1-a^{-1} q^{c_{3}-c_{1}}\right)(1-a)}{\left(1-q^{c_{1}}\right)\left(1-q^{c_{2}-c_{1}}\right)\left(1-q^{c_{3}-c_{1}}\right)\left(1-q^{c_{4}-c_{1}}\right)}, \\
& C=-\frac{a\left(1-a q^{c_{2}-c_{1}}\right)\left(1-a^{-1} q^{c_{3}-c_{2}}\right)(1-a)}{\left(1-q^{c_{2}}\right)\left(1-q^{c_{2}-c_{1}}\right)\left(1-q^{c_{3}-c_{2}}\right)\left(1-q^{c_{4}-c_{2}}\right)}, \\
& D=\frac{\left(1-a q^{c_{3}-c_{1}}\right)\left(1-a q^{c_{3}-c_{2}}\right)(1-a)}{\left(1-q^{c_{3}}\right)\left(1-q^{c_{3}-c_{1}}\right)\left(1-q^{c_{3}-c_{2}}\right)\left(1-q^{c_{4}-c_{3}}\right)}, \\
& E=\frac{\left(1-a q^{c_{4}-c_{1}}\right)\left(1-a q^{c_{4}-c_{2}}\right)\left(1-a q^{c_{4}-c_{3}}\right)}{\left(1-q^{c_{4}}\right)\left(1-q^{c_{4}-c_{1}}\right)\left(1-q^{c_{4}-c_{2}}\right)\left(1-q^{c_{4}-c_{3}}\right)}
\end{aligned}
$$

note that $A=C+E-B-D$.

Then by telescoping and converting the sums into the original notion and taking care again about the omitted factor, we obtain

$$
\begin{aligned}
& \sum_{t=1}^{n} \frac{U_{2 k\left(t+m_{1}\right)+2 m} U_{2 k\left(t+m_{2}\right)+2 m} U_{2 k\left(t+m_{3}\right)+2 m}}{P_{10}(W, \bar{W})} \\
& =\frac{\left(A^{2}-B^{2}\right) \alpha^{2 m+5}}{\Delta A^{6} B^{8}}\left(\frac{W_{2 k\left(m_{2}-m_{1}\right)}\left(B^{2}, A^{2}\right) W_{2 k\left(m_{3}-m_{1}\right)}\left(B^{2}, A^{2}\right)}{U_{2 k m_{1}} U_{2 k\left(m_{2}-m_{1}\right)} U_{2 k\left(m_{3}-m_{1}\right)} U_{2 k\left(m_{4}-m_{1}\right)}} T_{1}\right. \\
& +\frac{W_{2 k\left(m_{2}-m_{1}\right)}\left(A^{2}, B^{2}\right) W_{2 k\left(m_{3}-m_{2}\right)}\left(B^{2}, A^{2}\right)}{U_{2 k m_{2}} U_{2 k\left(m_{2}-m_{1}\right)} U_{2 k\left(m_{3}-m_{2}\right)} U_{2 k\left(m_{4}-m_{2}\right)}} T_{2} \\
& +\frac{W_{2 k\left(m_{3}-m_{1}\right)}\left(A^{2}, B^{2}\right) W_{2 k\left(m_{3}-m_{2}\right)}\left(A^{2}, B^{2}\right)}{U_{2 k m_{3}} U_{2 k\left(m_{3}-m_{1}\right)} U_{2 k\left(m_{3}-m_{2}\right)} U_{2 k\left(m_{4}-m_{3}\right)}} T_{3} \\
& \left.-\frac{\Delta W_{2 k\left(m_{4}-m_{2}\right)}\left(A^{2}, B^{2}\right) W_{2 k\left(m_{4}-m_{1}\right)}\left(A^{2}, B^{2}\right) W_{2 k\left(m_{4}-m_{3}\right)}\left(A^{2}, B^{2}\right)}{\left.\left(A^{2}-B^{2}\right) U_{2 k m_{4}} U_{2 k\left(m_{4}-m_{1}\right)} U_{2 k\left(m_{4}-m_{2}\right)} U_{2 k\left(m_{4}-m_{3}\right)}\right)} T_{4}\right),
\end{aligned}
$$


with $\alpha-\beta=\Delta$ and the sums

$$
T_{i}=\sum_{t=1}^{2 k m_{i}}\left(\frac{\alpha^{n+2 t k}}{W_{n+2 t k+2 m}\left(A^{2}, B^{2}\right)}-\frac{\alpha^{2 t k}}{W_{2 t k+2 m}\left(A^{2}, B^{2}\right)}\right) .
$$

4. ii) For $0<m_{1}<m_{2}<m_{3}<m_{4}<m_{5}$, we consider the sums

$$
\sum_{t=1}^{n} \frac{1}{P_{12}(W, \bar{W})} \text {. }
$$

If we convert it into $q$-notation and write $z=q^{2 t k}, q^{2 m}=c, q^{2 k m_{i}}=q^{c_{i}}$ and $(B / A)^{2}=a$ for $1 \leq i \leq 5$, then it equals

$$
\begin{aligned}
& \frac{(1-q)^{6}}{A^{12} \alpha^{2\left(6 m+k m_{1}+k m_{2}+k m_{3}+k m_{4}+k m_{5}-6\right)}} \\
& \times \sum_{t=1}^{n} \frac{z^{3}}{(1-a c z)\left(1-a c z q^{c_{1}}\right)\left(1-a c z q^{c_{2}}\right)\left(1-a c z q^{c_{3}}\right)\left(1-a c z q^{c_{4}}\right)\left(1-a c z q^{c_{5}}\right)} .
\end{aligned}
$$

Without constant factor, we consider the sums

$$
\begin{aligned}
S_{n} & :=\sum_{t=1}^{n}\left(\frac{z^{3}}{(1-a c z)\left(1-a c z q^{c_{1}}\right)\left(1-a c z q^{c_{2}}\right)}\right. \\
& \left.\times \frac{1}{\left(1-a c z q^{c_{3}}\right)\left(1-a c z q^{c_{4}}\right)\left(1-a c z q^{c_{5}}\right)}\right) .
\end{aligned}
$$

By partial fraction decomposition of the summand and using telescoping, we write

$$
\begin{aligned}
a^{3} c^{3} S_{n} & =-F \sum_{t=1}^{n}\left(\frac{1}{1-a c q^{c_{5}} z}-\frac{1}{1-a c z}\right) \\
& +E \sum_{t=1}^{n}\left(\frac{1}{1-a c q^{c_{4} z}}-\frac{1}{1-a c z}\right)-D \sum_{t=1}^{n}\left(\frac{1}{1-a c q^{c_{3} z}}-\frac{1}{1-a c z}\right) \\
& +C \sum_{t=1}^{n}\left(\frac{1}{1-a c q^{c_{2}} z}-\frac{1}{1-a c z}\right)-B \sum_{t=1}^{n}\left(\frac{1}{1-a c q^{c_{1}} z}-\frac{1}{1-a c z}\right)
\end{aligned}
$$

where

$$
\begin{aligned}
& A=\frac{1}{\left(1-q^{c_{1}}\right)\left(1-q^{c_{2}}\right)\left(1-q^{c_{3}}\right)\left(1-q^{c_{4}}\right)\left(1-q^{c_{5}}\right)}, \\
& B=\frac{q^{2 c_{1}}}{\left(1-q^{c_{1}}\right)\left(q^{c_{1}}-q^{c_{2}}\right)\left(q^{c_{1}}-q^{c_{3}}\right)\left(q^{c_{1}}-q^{c_{4}}\right)\left(q^{c_{1}}-q^{c_{5}}\right)},
\end{aligned}
$$




$$
\begin{aligned}
& C=\frac{q^{2 c_{2}}}{\left(1-q^{c_{2}}\right)\left(q^{c_{1}}-q^{c_{2}}\right)\left(q^{c_{2}}-q^{c_{3}}\right)\left(q^{c_{2}}-q^{c_{4}}\right)\left(q^{c_{2}}-q^{c_{5}}\right)}, \\
& D=\frac{q^{2 c_{3}}}{\left(1-q^{c_{3}}\right)\left(q^{c_{1}}-q^{c_{3}}\right)\left(q^{c_{2}}-q^{c_{3}}\right)\left(q^{c_{3}}-q^{c_{4}}\right)\left(q^{c_{3}}-q^{c_{5}}\right)}, \\
& E=\frac{q^{2 c_{4}}}{\left(1-q^{c_{4}}\right)\left(q^{c_{1}}-q^{c_{4}}\right)\left(q^{c_{2}}-q^{c_{4}}\right)\left(q^{c_{3}}-q^{c_{4}}\right)\left(q^{c_{4}}-q^{c_{5}}\right)}, \\
& F=\frac{q^{2 c_{5}}}{\left(1-q^{c_{5}}\right)\left(q^{c_{1}}-q^{c_{5}}\right)\left(q^{c_{2}}-q^{c_{5}}\right)\left(q^{c_{3}}-q^{c_{5}}\right)\left(q^{c_{4}}-q^{c_{5}}\right)} ;
\end{aligned}
$$

note that $A=F-E+D-C+B$.

Consequently, we obtain

$$
\begin{aligned}
& \sum_{t=1}^{n} \frac{1}{P_{12}(W, \bar{W})}=\frac{\alpha^{6} \Delta}{A^{6} B^{6}} \\
& \times\left(-\frac{1}{U_{2 k m_{5}} U_{2 k\left(m_{5}-m_{1}\right)} U_{2 k\left(m_{5}-m_{2}\right)} U_{2 k\left(m_{5}-m_{3}\right)} U_{2 k\left(m_{5}-m_{4}\right)}} T_{5}\right. \\
& +\frac{1}{U_{2 k m_{3}} U_{2 k\left(m_{4}-m_{1}\right)} U_{2 k\left(m_{4}-m_{2}\right)} U_{2 k\left(m_{4}-m_{3}\right)} U_{2 k\left(m_{5}-m_{4}\right)}} T_{4} \\
& -\frac{1}{U_{2 k m_{3}} U_{2 k\left(m_{3}-m_{1}\right)} U_{2 k\left(m_{3}-m_{2}\right)} U_{2 k\left(m_{4}-m_{3}\right)} U_{2 k\left(m_{5}-m_{3}\right)}} T_{3} \\
& +\frac{1}{U_{2 k m_{2}} U_{2 k\left(m_{2}-m_{1}\right)} U_{2 k\left(m_{3}-m_{2}\right)} U_{2 k\left(m_{4}-m_{2}\right)} U_{2 k\left(m_{5}-m_{2}\right)}} T_{2} \\
& \left.-\frac{1}{U_{2 k m_{1}} U_{2 k\left(m_{2}-m_{1}\right)} U_{2 k\left(m_{3}-m_{1}\right)} U_{2 k\left(m_{4}-m_{1}\right)} U_{2 k\left(m_{5}-m_{1}\right)}} T_{1}\right),
\end{aligned}
$$

where the sum $T_{i}$ is defined as before.

Of course, we could invent many more examples, but we think that the message is clear now.

It should be noted that our elementary method can always be used to simplify sums of the type considered here; even if they do not telescope, they lead to simpler answers.

\section{REFERENCES}

[1] R. Melham, "Finite reciprocal sums involving summands that are balanced products of generalized Fibonacci numbers." J. Integer Seq., vol. 17, no. 6, pp. article 14.6.5, 11, 2014. 
Authors' addresses

\section{E. Kılıç}

TOBB University of Economics and Technology, Mathematics Department, 06560 Ankara, Turkey

E-mail address: ekilic@etu.edu.tr

H. Prodinger

Department of Mathematics, University of Stellenbosch, 7602 Stellenbosch, South Africa

E-mail address: hproding@sun.ac.za 